\title{
Image Segmentation of Color Image Based on Region Coherency
}

\author{
S. Ji and H.W. Park \\ Dept. of Electrical Engineering \\ Korea Advanced Institute of Science and Technology \\ Taejon, Korea \\ hwpark@athena.kaist.ac.kr
}

\begin{abstract}
Two-step image segmentation algorithm is proposed, which is based on region coherency for the segmentation of color image. The first step is the watershed segmentation, and the next one is the region merging using artificial neural networks. Spatially homogeneous regions are obtained by the first step, but the regions are oversegmented. The second step merges the oversegmented regions. The proposed method exploits the luminance and chrominance difference components of color image to verify region coherency. The YUV color coordinate system is used in this work.
\end{abstract}

\section{Introduction}

Image segmentation is very useful method for efficient data compression in MPEG-4 and the coming standard MPEG-7. Efficient segmentation of intra- and inter-images is important to reduce data amount optimally in conjunction with shape coding and texture coding. Image segmentation using the only luminance component of the color image is very difficult task. Pappas [1] considered the problem of segmenting images with smooth surfaces, which presents a generalization of the K-means clustering algorithm to include spatial constraints and to account for local intensity variations in the image. Vincent [2] introduced a fast and flexible algorithm for computing watersheds in digital grayscale images. Najman [3] proposed the notion of dynamics of a minimum, which is based on geodesic reconstruction to prevent the oversegmentation of the watershed.

An intra-image segmentation method is proposed in this paper, which consists of watershed segmentation and region merging by using luminance, chrominance, and area constraints. To perform the intra-image segmentation of color image, two-step algorithm is proposed. In the first step, the watershed segmentation is applied to the luminance component of the color image. But the result is the oversegmented image. To reduce the oversegmentation of the watershed algorithm, the artificial neural networks are used. As inputs to the neural networks, the area of the watershed segments, the chrominance components and the luminance component are used.

In this paper, section 2 describes the proposed algorithm, which consists of the watershed algorithm and region merging algorithm. Section 3 shows the experimental results, and is followed by conclusion in section 4 .

\section{Algorithm}

\subsection{Watershed segmentation}

The first step of the proposed algorithm is the watershed segmentation [2]. The watershed algorithm is performed on the gradient image of the luminance component, where the gradient image is filtered by the morphological grayscale opening before the watershed algorithm. The morphological opening simplifies the original signal (the gradient image) by removing the bright components that do not fit the structuring elements, which is defined as follows,

$$
\begin{aligned}
& \gamma(f)=\delta(\varepsilon(f)) \\
& \text { where } \\
& \varepsilon(f)(x)=\min \{f(x+y), y \in D\} \\
& \delta(f)(x)=\max \{f(x-y), y \in D\}
\end{aligned}
$$

In eq. (1), $f(x)$ denotes an input signal, $D$ is a window 
or structuring element, $\delta$ is the morphological dilation, $\varepsilon$ is the morphological erosion, and $\gamma$ is the morphological opening.

The watershed algorithm uses the gradient of gray level for segmentation, so that it can segment image into several homogeneous regions which has the same or similar gray levels. In order to perform meaningful segmentation of image, regions of different gray levels should be merged if the regions are from the same object. The watershed segmentation generates spatially homogeneous regions, which are oversegmented. The watershed is often used in conjunction with the geodesic reconstruction, which simplifies the gradient images and prevents the oversegmentation [4]. Those oversegmented regions should be merged via region coherency so that the segmentation could be meaningful as correctly as possible.

\subsection{Merge}

The second step is the region merging. The merging strategies are based on the information of the luminance, the chrominance difference, the region proximity, and the areas of the watershed regions. The luminance component is usually used for the segmentation of the image, but it brings out some problem because it cannot separate two semantically or physically different objects with the same or similar luminance values. So the chrominance components can be used as additional information for the optimum segmentation.

In YUV color coordinates system that is used in MPEG standards, $U$ and $V$ are two chrominance components. The YUV color coordinates system is used efficiently for image compression, which is defined as follows,

$$
\begin{aligned}
& Y=0.3 R+0.6 G+0.1 B \\
& U=Y-B \\
& V=Y-R
\end{aligned}
$$

In order to utilize chrominance components for image segmentation, we define the chrominance difference $\mathrm{C}$ $=\mathrm{V}-\mathrm{U}$, which is also orthogonal to the luminance component $\mathrm{Y}$ and can approximately represent the illumination variation.
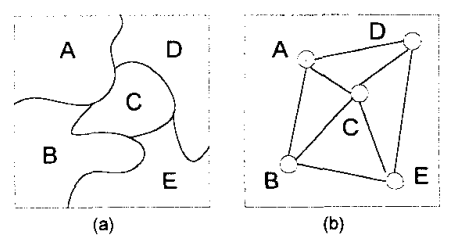

Fig. 1 Segmented regions and adjacency graph
To merge watershed regions, they are arranged with the neighborhood graphs and adjacency graphs by the graph theory. Figure 1 shows an example of the segmented image and its adjacency graph.

The Kohonen self-organizing map (SOM) in Fig. 2 is used for region merging with merging criteria of three dimensional feature vector $\vec{F}_{3}=\left(Y_{\text {mean }}, C_{\text {mean }}, A\right)$, where the first component $Y_{\text {mean }}$ is the mean of luminance of each region, the second component $C_{\text {mean }}$ is the mean of chrominance difference of each region, and the last component $A$ is the area of each segmented region.

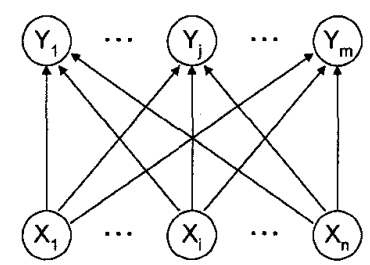

Fig. 2 Kohonen self-organizing map (SOM)

The merging strategy has three different phases whose flowchart is shown in Fig. 3. The first phase uses SOM with two dimensional feature vectors $\vec{F}_{2}=\left(Y_{\text {mean }}, C_{\text {mean }}\right)$, the second phase uses SOM with the three features of the feature vector $\vec{F}_{3}$, and the last phase merges the small area regions into the larger area regions. The watershed regions start to gather into clusters at the first phase.

The characteristic of the clustering process using SOM is as follows: When choosing the winner, instead of using simple Euclidean distance between feature vector and weight vector, Euclidean distance is weighted by the cumulative frequency of occurrences (Frequency Sensitive Competitive Learning). The second winner is repelled by the winner (Rival Penalized Competitive Learning) [5]. Learning rate $\eta$ is calculated from within-cluster variances $v_{i}(3),[6]$.

$$
\eta=\frac{\ln (K)-H\left(v_{1}, v_{2}, \ldots, v_{K}\right)}{\ln (K)}
$$

where

$$
\begin{aligned}
& H\left(v_{1}, v_{2}, \ldots, v_{K}\right)=\sum_{k=1}^{K}-v_{k, \text { norm }} \ln \left(v_{k, \text { norm }}\right) \\
& v_{k, \text { norm }}=v_{k} / \sum_{i=1}^{K} v_{i}
\end{aligned}
$$

where $\mathrm{K}$ is the number of clusters.

All the regions within the same cluster cannot be merged. Only the regions that satisfy the region proximity can be merged together. The region 
proximity is easily inferred by the neighborhood and adjacency graphs. Using the feature vector and the proximity constraint, only spatially coherent regions can be merged with fidelity.

The region merging is performed by three kinds of conditional loops starting from the merging phase 1 in Fig. 3 as discussed above. In the first loop, when the resulting number of the merged regions of the previous stage is below a threshold $T h_{2}$, the first phase of the merging strategy proceeds, whereas the third phase of the merging strategy proceeds when the resulting number is above $T h_{2}$.

When the number of the merged regions of the first loop is below $T h_{1}$, the second conditional loop operates until the resulting number is below a threshold $T h_{3}$. In this loop, the second phase of the merging strategy proceeds when the number of merged regions is below $T h_{2}$, but the third phase of the merging strategy proceeds when the resulting number is above $T h_{2}$. When the second phase of the merging strategy proceeds, the area constraint is critical. The first phase of the strategy treats only small area regions that are not important semantically or physically. On the contrary, in the second phase of the strategy, we treat large area regions as well as small area regions. In this case, the proposed algorithm considers the region coherency, so that two large area regions can be prevented from being merged together.

At last, when the number of the merged regions of the second loop is below $T h_{3}$, the third phase of the merging strategy proceeds with the third loop until it reaches the stopping condition. In this phase, only small area regions that are not important semantically or physically are merged into larger regions.

\section{Experimental Results}

The proposed algorithm is applied to a test image of $0^{\text {th }}$ frame in "Mother and Daughter Sequence" which has resolution of QCIF as shown in Fig. 4. Figure 4 (b) is the result of watershed segmentation, which is oversegmented and the segments have no semantic meaning. Figure 4 (c) is the merged segments by the proposed algorithm. The final segments have good semantic and physical meaning.

In this case, the watershed segmentation in Fig. 4 (b) has 272 regions. The first conditional loop iterates 3 times and results in 52 regions. The second conditional loop iterates once and results in 28 regions. Finally, the third conditional loop iterates 4 times and results in 19 regions. So 272 regions reduce to 19 regions. In Fig. 4, the face, hair, and body of mother are successfully segmented. The head and body of children are successfully segmented, too.

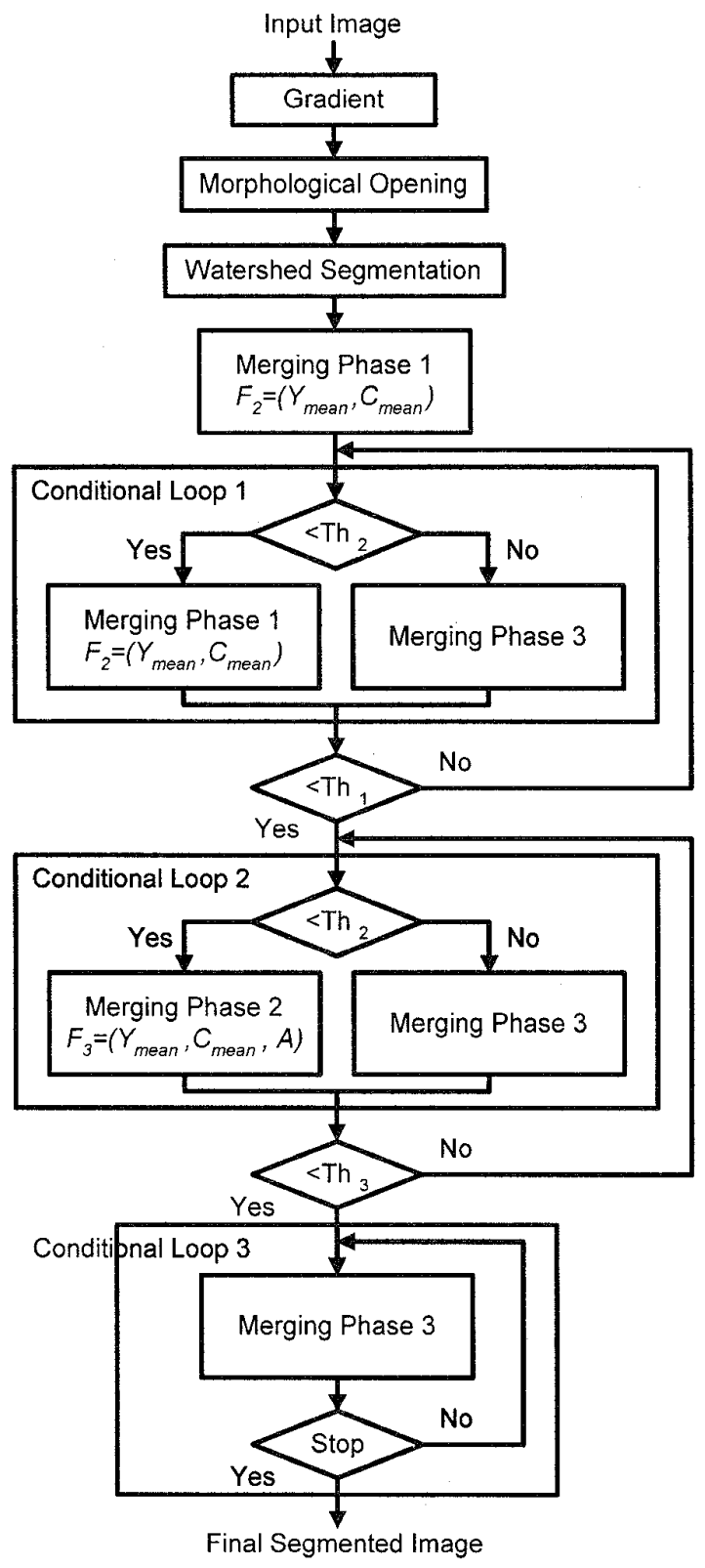

Fig. 3 Flowchart of the proposed segmentation algorithm

Total computation time of one frame of QCIF color image is about 3 seconds at PC (Pentium 90MHz CPU and 64MBytes memory). It is too long for real-time processing. We will continue to study this work to reduce the computation time and to obtain better segmentation. 


\section{Conclusions}

To perform the image segmentation of intra-image, an image segmentation method is proposed, which uses the chrominance difference, the luminance component, the area constraint, and the region proximity. The proposed method considers that two regions with similar or the same luminance components but semantically or physically different properties cannot be merged together with safety during region merging.

\section{References}

[1] T. N. Pappas, "An adaptive clustering algorithm for image segmentation," IEEE Trans. on Signal Processing, Vol. 40, No. 4, April 1992

[2] L. Vincent and P. Soille, "Watersheds in Digital Spaces:
An Efficient Algorithm Based on Immersion Simulations," IEEE Trans. on PAMI, Vol. 13, No. 6, June 1991

[3] L. Najman and M. Schmitt, "Geodesic saliency of watershed contours and hierarchical segmentation," IEEE Trans. on PAMI, Vol. 18, No. 12, December 1996

[4] P. Salembier and M. Pardas, "Hierarchical Morphological Segmentation for Image Sequence Coding," IEEE Trans. on Image Processing, Vol. 3, No. 5 September 1994

[5] L. Xu, A. Krzyzak, and E. Oja, "Rival Penalized Competitive Learning for Clustering Analysis, RBF Net, and Curve Detection," IEEE Trans. on Neural Networks, Vol. 4, No. 4, July 1993

[6] C. Chinrungrueng and C. H. Sequin, "Optimal Adaptive K-Means Algorithm with Dynamic Adjustment of Learning Rate," IEEE Trans. on Neural Networks, Vol. 6, No. 1, January 1995

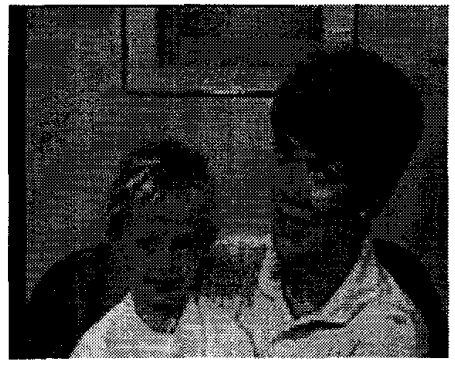

(a)

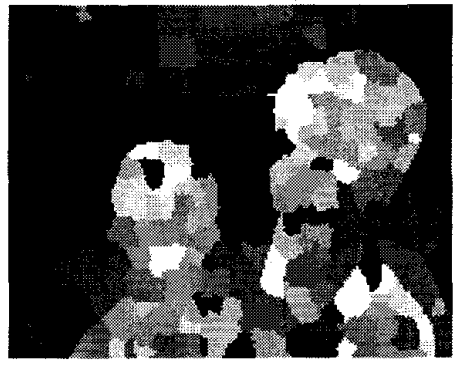

(b)

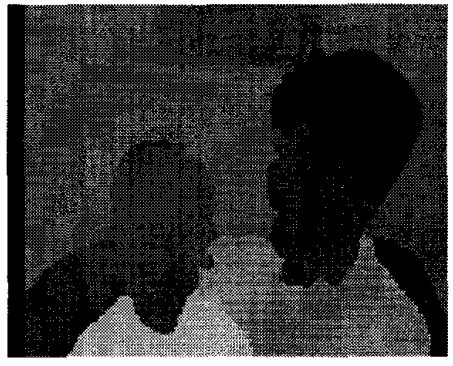

(c)

Fig. 4 An example of the segmentation

(a) Original $0^{\text {th }}$ fame image of mother and daughter (QCIF), (b) the result of watershed segmentation, and

(c) final segmented image from the proposed method 